\title{
It from Bit from It from Bit... Nature and Nonlinear Logic
}

\author{
Wm. C. McHarris \\ Departments of Chemistry and Physics/Astronomy \\ Michigan State University \\ East Lansing, MI 48824
}

"Quantum mechanics is the very epitome of a linear science."

"Before observation a system can exist in a superposition of quantum states, such as Schrödinger's cat's being both dead and alive."

"A particle such as an electron is intrinsically neither particle nor wave but exists in an epistemological limbo: if one performs an experiment to measure particle-like properties, it cooperates and acts as a particle; if one seeks wave-like properties, it behaves as a wave."

"Classical mechanics is a meso- or macro-scale approximation to quantum mechanics, with the Correspondence Principle connecting the two."

"The experimental violations of Bell-type inequalities demonstrate that local reality fails and entangled particles can communicate faster than the speed of light - contrary to the laws of relativity. Einstein's 'spooky action at a distance' does in fact occur."

For many decades statements such as these formed something akin to a physicist's bible. Despite the many paradoxes that resulted from this orthodox Copenhagen interpretation, few dared to doubt its validity. The holdouts, such as Einstein, Schrödinger, deBroglie, and later Bohm et al., were considered to be past their prime - after all, Bohr had vanquished Einstein in their debates and in his response to the EPR paper of 1935 [1]. Reductionism reigned supreme!

Then along came quantum information science with the enticement of super-fast quantum computing. Interest revived in the fundamentals of quantum mechanics - now and then people began to question tenets of the Copenhagen interpretation and to proffer alternatives. There were even a few attempts to deal with possible nonlinearities intrinsic to quantum mechanics, but these were treated as perturbations on a basically linear theory [2], a situation in which extreme characteristics of nonlinear dynamics, demonstrated by systems in or at the edge of chaos, cannot come to pass [3]. Strongly nonlinear systems, in which such characteristics are allowed to develop, can seem most peculiar - indeed, they can appear to be every bit as counterintuitive and paradoxical as quantum mechanics itself. However, just as with the oddities of relativity, upon more detailed examination these nonlinear peculiarities are discovered to be considerably more logical than the paradoxes of orthodox quantum mechanics. 
During the past decade I have published a number of papers in which it was demonstrated that many of the so-called paradoxes generated by the Copenhagen interpretation of quantum mechanics have parallels in nonlinear dynamics and chaos $[4,5$, and references therein]. For example, the inherent randomness of radioactive decay can be reconciled with observed first-order exponential decay laws through the extreme (exponential) dependence on initial conditions - the "Butterfly Effect." Or, chaotic scattering of classical particles can produce diffraction-like patterns such as those characteristic of waves [6]. And strongly nonlinear classical systems can exhibit correlations - or entanglements! - analogous to those of quantum systems, thereby violating Bell-type inequalities [4, 7]; also, nonergodic behavior can easily ape "action at a distance." Indeed, macroscopic nonlinear systems produce quantization intrinsically, for they often organize themselves into "quantized" or resonant levels, which are governed by eigenvalue equations analogous to the Schrödinger equation.

These parallels and analogies by no means prove that quantum mechanics is fundamentally nonlinear, and this is not the forum in which to delve into their detailed justification. Nevertheless, they do raise important, fundamental questions about the possibilities of strongly nonlinear effects at the heart of quantum mechanics. Strongly nonlinear and/or chaotic systems can be deterministic, i.e., a given set of initial conditions leads deterministically to a specific final state - cause and effect - but because it is normally impossible to determine the initial conditions with sufficient precision to achieve such a prediction, such systems must be treated statistically. Deterministic chaos provides the determinism so dear to Einstein, yet it must be treated statistically à la Bohr and the Copenhagen School. Perhaps it can provide the bridge between the two viewpoints - in hindsight, both Einstein and Bohr could have been correct in their debates!

During the past fifteen or so years I have given close to fifty lectures on some variation of "Chaos and the Quantum" at universities, national laboratories, and regionalnational-international conferences - some to physicists studying the fundamental bases of quantum mechanics, others to sundry scientists studying nonlinear dynamics, chaos, and complexity. I have found very little communication between the two groups: Nonlinear dynamicists know very little about the fundamentals of quantum mechanics, and while physicists may have heard of nonlinear dynamics and chaos, they mostly consider them to lie in an obscure corner of science, with little relevance to their primary goals. At times I have felt as if I had one foot resting on a quantum ice-floe, the other on a chaotic ice-floe - while the two are rapidly drifting apart!

A favorite quip of nonlinear dynamicists is, "Calling most of dynamics nonlinear dynamics is like calling all of zoology not concerned with elephants nonpachydermology!" Most of nature is indeed nonlinear, filled with various forms of feedback. Nonlinear dynamics and chaos, although a new science, has rapidly gained enormous success in fields as diverse as biology, chemistry, economics, even traffic patterns. The glaring exception has been quantum mechanics, which should make us suspicious. I feel that non- 
linear dynamics and chaos theory are changing not only the way we do science, but also the way we view the world (and the universe). Yet most of us have difficulty in thinking nonlinearly or dealing with nonintuitive feedback, just as we have difficulty in coping with limits such as infinity or correlated, Bayesian statistics. Since this is primarily a physics forum, I limit myself to two straightforward, intentionally over-simplified demonstrations of nonlinear dynamics, illustrating nonlinear logic.

\section{Nonlinear analysis.}

We are simply not used to thinking in terms of nonlinear logic. Sure, we can deal with simple systems. Take, for example, the deer population in Northern Michigan. A typical environment can only support so many deer. As the population increases, the deer overgraze, causing the forage to decrease, which eventually leads to less healthy, then fewer deer. With fewer deer, the vegetation recovers, which leads again to more deer. A straightforward example of feedback. We would not be surprised to find a herd oscillating between, say, fifty and twenty deer in alternate years. And, with climate change making Northern Michigan more verdant, we could easily understand a trend that saw the same herd increase and oscillate between larger numbers, such as eighty and thirty-two deer. It would merely be a case of different environmental parameters.

But suppose we performed a carefully controlled study and discovered that a given herd oscillated over a longer timespan among four distinct numbers, or even eight numbers - or that the number of deer became completely unpredictable even though we were working under carefully controlled conditions? I wager that such an analysis would discourage most of even the hardiest researchers. This, however, is a relatively simple case of biological feedback, where nonlinear dynamics, then chaos sets in. And we shall see in the next section that it can be explained by a simple model. Chaos is the situation where seemingly complex, even inexplicable behavior results from simple systems.

Another straightforward example that confounds most people is the mixing - or unmixing - of powders in a nonlinear tumbler, a rotating cylinder having part of one arc replaced by a flattened side [8]. A mixture of salts often separates into distinct bands in such a tumbler. Surely this is a spontaneous decrease in entropy, contrary to everything we have learned abut the Second Law of Thermodynamics?! Yet it happens. An even simpler demonstration (gleaned from the same article) is the following: Fill a cylindrical bottle, about $5 \mathrm{~cm}$ in diameter x 15-20 cm long, roughly $60 \%$ full of common $\mathrm{NaCl}$. Then add a plastic map tack and an iron hexagonal nut. When you shake the bottle horizontally one of the two comes to the top, but vertically, the other. I leave it to you to determine which is which. A simple-minded demonstration, but one that makes a strong impression on college chemistry students.

A less pleasant onset of chaos can occur when a complicated system under construction has its end goals altered. This has occurred on at least two occasions with large, new computer systems. Both the U.S. Red Cross Blood Bank software operating system 
and one of the U.S. Naval Defense systems suffered this fate, when succeeding administrations changed the ultimate goals of the software numerous times. Chaos set in, and the systems became unmanageable and unusable and had to be replaced almost from scratch, at considerable financial loss.

Perhaps the most dramatic illustrations of how little we comprehend nonlinear logic arise in computer science from evolutionary computer programs. Initiated at MIT and Stanford, this sort of research is now being performed at many major universities, including MSU. Here is a seemingly straightforward example.

A popular yardstick among computer scientists is to create an efficient program that will sort, say, a set of 100 random numbers into ascending order. To simulate evolution in creating such a program, one first uses a pseudorandom number generator to generate programs consisting of (almost) random sequences of numbers. One can then either use these programs "raw," or to speed up the process, one can retain only instructions at least marginally useful for sorting, such as comparison and exchange instructions. Thus, one begins with a population consisting of, say, 10,000 random programs, each consisting of several hundred instructions.

One then runs and tests these randomly generated programs, which, as expected, do a very poor job at first. Only the "most fit," meaning any programs that show the slightest inclination for sorting, are retained. These are used to create the next generation. This can be done in two ways: First, by inserting random minor variations, corresponding to asexual mutations; second, by "mating" parent programs to create a child program, i.e., by splicing parts of programs together, hoping that useful instructions from each parent occasionally will be inherited and become concentrated. This process can be repeated thousands upon thousands of time with fast parallel processors, and eventually very efficient programs can result. (It should be mentioned in passing that this sort of research is carried out on isolated computers using nonstandard operating systems, for such programs could become dangerous viruses on the internet.)

Some of the results were startling. Danny Hillis, who originated many aspects of these programs, sums it up vividly in his book, The Pattern on the Stone [9]:

I have used simulated evolution to evolve a program to solve specific sorting problems, so I know that the process works as described. In my experiments, I also favored the programs that sorted the test sequences quickly, so that faster programs were more likely to survive. This evolutionary process created very fast sorting programs. For the problems I was interested in, the programs that evolved were actually slightly faster than any of the algorithms described ...[standard algorithms].- and, in fact, they were faster at sorting numbers than any program I could have written myself.

One of the interesting things about the sorting programs that evolved in my experiment is that $I$ do not understand how they work. [my emphasis] I have carefully examined their instruction sequences, but I do not understand 
them; I have no simpler explanation of how the programs work than the instruction sequences themselves. It may be that the programs are not understandable that there is no way to break the operation of the program into a hierarchy of understandable parts. If this is true - if evolution can produce something as simple as a sorting program which is fundamentally incomprehensible - it does not bode well for our prospects of ever understanding the human brain.

This evolutionary process has also been used to design electronic circuits [10], with similar results. Circuits can result that are considerably more efficient than those produced by professional designers. Sometimes they also contains superfluous appendages, apparently leftovers from the evolutionary process. But again, they cannot be analyzed in any straightforward manner. They just work. It should also be mentioned that in all of these processes there is a tendency to reach only local maxima. The introduction of "predator" programs overcomes this, forcing the attainment of global maxima - completely analogous to Darwinian evolution.

In other words, there are logical processes that cannot be understood, much less be broken down into reductionist, simply analyzable parts. On the positive side, nature is far more intricate and beautiful than we could imagine, using our simple(-minded) models. On the negative side, nonlinear systems tend not to be generalizable (despite Feigenbaum's Universality in chaotic systems). Thus, producing, say, a Boolean system of analyzing nonlinear logic may be beyond us.

\section{Infinite Regression and Free Will.}

Extreme, exponential sensitivity to initial conditions in deterministic chaotic systems means just that - although a given set of initial conditions most certainly leads to a definite final state at a specified future time, in practice it is impossible, even unthinkable, to determine a set of initial conditions with anywhere nearly enough precision in order to produce a predetermined final state. (In practice, chaoticists get around this presumed limit to the usefulness of chaos theory because other chaotic orbits in phase space "shadow" the desired orbit, making statistical predictions possible. But this is beyond the scope of this essay.) A simple example illustrates this:

Consider a simple three-dimensional iron-bob pendulum free to oscillate above a surface that contains three magnets arranged in a triangle - see Fig. 1. We color the "basins of attraction" of these three magnets red, blue, and green. That is, if the pendulum is released somewhere above the large red blob, it will eventually wind up near the "red" magnet. (Although in this instance the magnets really do attract, the term "basin of attraction" refers to any region in phase space that "surrounds" a point where an orbit is likely to wind up, whether or not the "attraction" is physical.) Similar behavior for the 


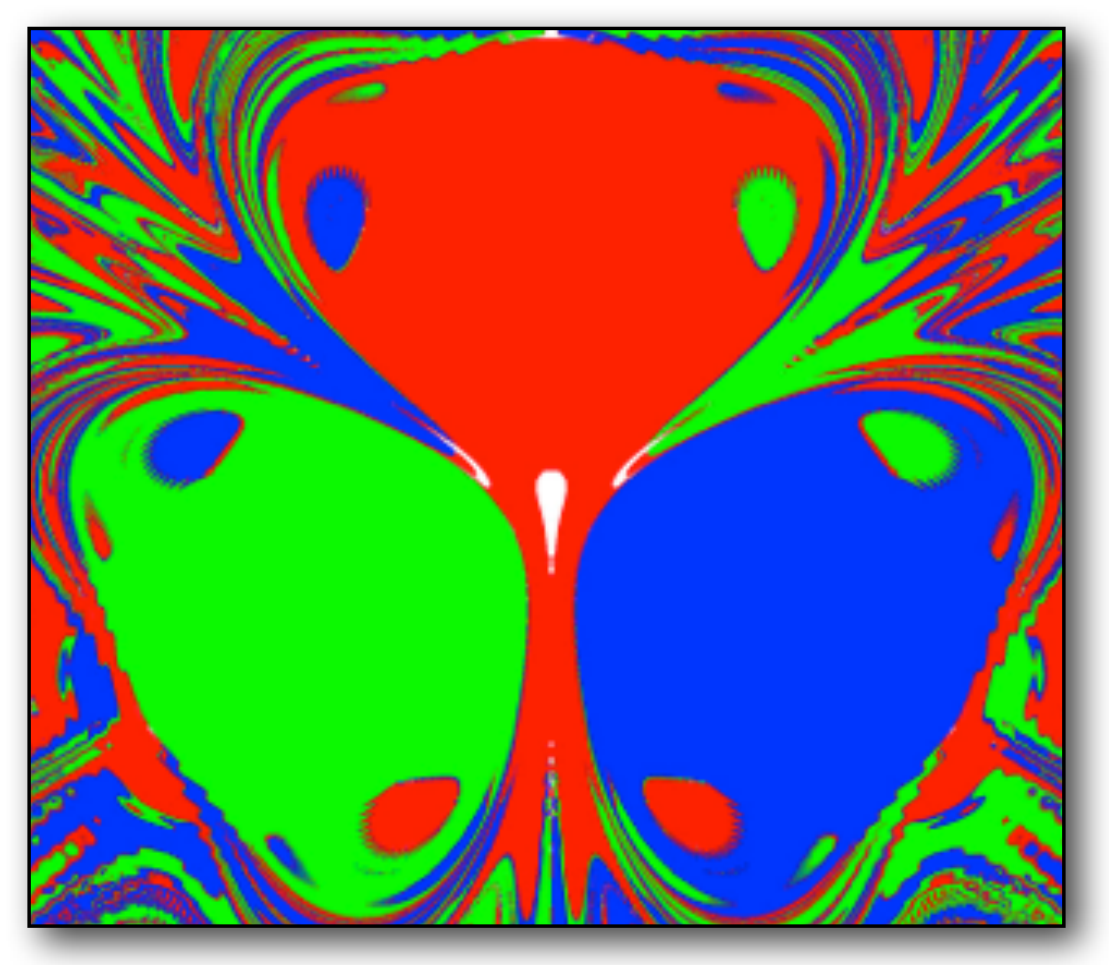

Fig. 1. Basins of attraction for a pendulum suspended above three magnets arranged in an equilateral triangle with the "red" magnet situated near the top of the red basin and "blue" and "green" magnets in corresponding positions on their basins. It can be seen that between every two colors lies a a basin of the third color, ad infinitum. Thus, there are regions where it is physically impossible, no matter how precise the experiment, to predict (other than statistically) where the pendulum will stop. This is an example of the Butterfly Effect.

"blue" and "green" magnets. But what if it is released near the boundary of the red and green basins - it very likely will wind up above the blue magnet. However, things get complicated very quickly. It has been shown that between every two colors there is a basin of the third color, ad infinitum. There are regions where it is physically impossible, no matter how great the precision of one's experiment, to predict where the pendulum will come to rest. This is true whether the pendulum is a toy as illustrated or a magnificent Foucault pendulum suspended from a many-storied dome. It just takes longer to reach the limit of precision with the Foucault pendulum - we say it takes longer for the uncertainty to catch up with and overwhelm the precision of the measurement. Intricately intwined basins of attraction such as these are known as "riddled basins," and they are the norm rather than the exception. (This is very similar to Newton's method for extracting the complex roots of a third-order polynomial, where there are regions of initial guesses that lead to unexpected roots. The basins of attractions look quite similar, an example of so-called Universality in chaos, where identical or very similar equations can be used to describe quite disparate situations)

Although the above example is quite simple to visualize, its math is not the sim- 
plest, so, in keeping with Occam's Razor, here is the very simplest, most studied example of infinite regression, the logistic map. Its history in describing population dynamics goes all the way back to the Belgian mathematician, Verhulst, in the 1840's.

The very simplest model for predicting the next generation population would be to multiply this generation's population $x_{n}$ by the birth rate $A$. However, if the birth rate is greater than 1, this leads to Malthusian, exponential growth, clearly unrealistic. One then notes that a given environment can only support so many individuals and includes the difference between this maximum and the present population as the first correction. The next generation is thus predicted to be

$$
x_{n+1}=A x_{n}\left(1-x_{n}\right) .
$$

What could be simpler and more predictable? However, as can be seen in Fig. 2, this equation can produce some rather unexpected behavior.
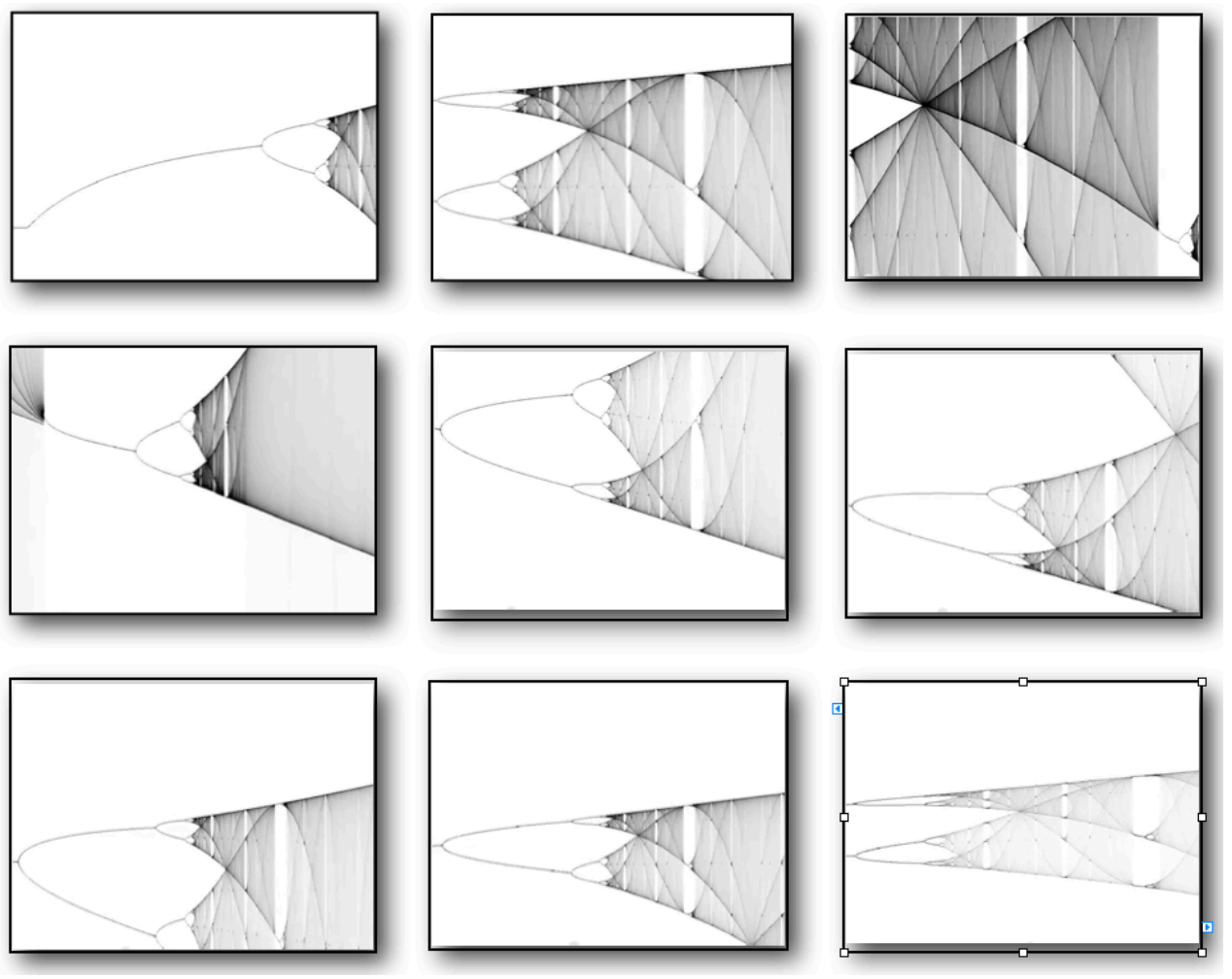

Fig. 2. Bifurcation diagram for the logistic map. Here the value of the final population, ranging from 0 (extinction) to 1 (maximum), is plotted against the control parameter (birth rate) $A$, which ranges from $<1$ to 4 (above 4 the model breaks down). The first frame shows the complete diagram, and each successive frame enlarges the point of interest nearest the middle by a factor of roughly 5 . 
First of all, for $A<1$, the population vanishes, as expected, for all initial values. (We say that here 0 is an attractor of the system.) For $A>1$, after sufficient iterations the population settles down to a single value that generally increases as $A$ increases, again as expected. Just above $A=3$ the final value bifurcates, i.e., alternates between two stable values. As $A$ continues to increase, further bifurcations occur, with periods of $4,8,16, \ldots$ (The periods are not simply powers of 2 but incur all the natural numbers in an elaborate number-theoretical sequence called the Sarkovskii ordering.) Finally, at $A>3.44948 \ldots$ chaotic behavior ensues, in which the map never settles down but continues hitting seemingly random values ad infinitum. The "dust" in Fig. 2 results not from poor graphics nor from not carrying out sufficient iterations to fill the gaps - it results from infinitely complex detail in the diagram. As can be seen from the successive magnifications, this diagram is said to be "self-similar" (or more correctly, "self-affine," because the frames are not exact copies). In the limit of infinite iterations, the magnification - no matter how high, even infinite - of any portion of the diagram resembles the diagram as a whole.

Now, if this chaotic behavior were truly random, there would be little point in following through with chaos theory. However, there is a definite, albeit subtle order in chaos. One of the clearest manifestations of this can be seen as the gaps in Fig. 2; one that stands out is the large period-3 gap in the vicinity of $A=3.82$. There are an infinite number of such gaps, persisting all the way down to infinite magnification. This means there are regions of order (periodic behavior) intimately mixed in with the chaotic regions. (To revert to quantum mechanics, although I promised I wouldn't, this could be analogous to duality, where periodic regions represent particle-like behavior, chaotic regions, wave-like behavior.)

But what does all of this mean in terms of free will? Simply this: Just as chaos theory can provide a bridge between determinism and statistical behavior, so can it provide a bridge between predestination and free will. Maps having self-affine infinite regression are ubiquitous in the universe, and these maps can be strictly deterministic - a given initial point inevitably leads to a definite final point. Nevertheless, because it is physically impossible to determine this initial point with the necessary infinite precision, one cannot work backward from the "predestined" final point to its defined cause. Mathematics can state things with certainty; physics cannot. In physics we are fond of stating, "In principle, it can be shown (even proven) that..." In principle, chaos theory shows this not to be true. Principle and practice represent two antipodal worlds - perhaps ne'er the twin shall meet.

Free will has received considerable attention in the fundamentals of quantum mechanics world in the last few years, in particular with the Free Will Theorem of Conway and Kochen [11], which interjects the idea of free will into the behavior of fundamental particles (at the Planck scale). It has been used to castigate certain contemporary interpretations of quantum theory. Could this not be necessary? Could free will be represented by a simpler (remember Occam's Razor) nonlinear infinite regression? 


\begin{abstract}
Afterword.
It from Bit or Bit from It? Nonlinear dynamics and chaos theory shows us that disparate parts of nature are intimately linked together much more tightly than we could previously have imagined. Wherever there is feedback there is crossover. We could well be fooling ourselves with our "straightforward" linear, reductionist models. Could it be significant that chaos theory has had successes in almost every scientific field other than quantum mechanics - to the extent that for decades respectable scientists have doubted the very existence of quantum chaos [12]. Could it be that quantum mechanics already contains essential nonlinear elements, and that when we try to apply chaos theory to it, we are going around in a loop? An esthetic advantage of injecting nonlinear dynamics into quantum mechanics is that it is relatively simple and straightforward (Occam's Razor, for the last time), does not lead us into the quagmire of assuming hidden variables (!), and it makes the transition from quantum to classical mechanics smoother, eliminating part of the problem of determining the border between observer and observed. Nonetheless, despite avowed pronouncements from a myriad of respected and respectable physicists, beauty in equations does not make a theory true — or relevant. Only experimental investigation - and the ability of a theory to be falsifiable can do that, which is why we have a significant number of expositions such as The Trouble with Physics nowadays [13].

As for information science: "It from Bit or Bit from It?" is a bit like the problem of the chicken and the egg. One can easily get trapped in an infinite loop. Which brings us back to where we started. It from Bit from It... involves nonlinearity and feedback, which leads us to nonlinear dynamics and chaos, which leads to infinite regressions, which leads to...
\end{abstract}




\section{References:}

[1] A. Einstein, B. Podolsky, and N. Rosen, Phys. Rev. 44, 777 (1935).

[2] S. Weinberg, Ann. Phys. (NY), 194, 336 (1989); N. Gisin, Phys. Lett. A143, 1 (1990); B. Mielnik, Phys. Lett. A289, 1 (2001).

[3] M. J. Feigenbaum, "Foreword" to Chaos and Fractals: New Frontiers of Science, 2nd Ed., H.-O. Peitgen, H. Jürgens, and D. Saupe, p. 1 (New York: SpringerVerlag, 2003).

[4] Wm. C. McHarris, J. Phys.: Conf. Ser. 306, 012050 (2011).

[5] Wm. C. McHarris, Complexity, 12(4), 12 (2007).

[6] S. Bleher, C. Grebogi, and E. Ott, Physics D, 46, 87 (1990); J.V. José and E.J. Saletan, Classical Dynamics: A ContemporaryApproach, Chap. 4, §4.1.3 (Cambridge: Cambridge University Press, 2002).

[7] M. Gell-Mann and C.J. Tsallis, eds., Nonextensive Entropy: Interdisciplinary Applications (New York: Oxford University Press, 2--4).

[8] T. Shinbrot and F.J. Muzzio, Physics Today, 53(3), 25 (2000).

[9] W.D. Hillis, The Pattern on the Stone: The Simple Ideas That Make Computers Work (New York: Basic Books, 1998).

[10] J.R. Koza, M.A. Keane, and M.J. Streeter, Sci. Am. Feb. 2003, p. 52.

[11] J. Conway and S. Kochen, Found. Phys. 36 (10), 1441 (2006).

[12] J. Ford, in The New Physics, P. Davies, Ed., Chap. 12 (Cambridge: Cambridge University Press, 1989).

[13] L. Smolin, The Trouble with Physics: The Rise of String Theory, the Fall of a Science, and What Comes Next (Boston: Houghton Mifflin Harcourt, 2006); P. Woit, Not Even Wrong: The Failure of String Theory \& the Continuing Challenge to Unify the Laws of Physics (New York: Basic Books, 2006). 